\title{
Sarcopenic Obesity: Correlation with Clinical, Functional, and Psychological Status in a Rehabilitation Setting
}

\author{
Lorenzo M. Donini ${ }^{*}$, Eleonora Poggiogalle' ${ }^{1}$, Silvia Migliaccio², Alessandro Pinto1, \\ Carla Lubrano' ${ }^{1}$, Andrea Lenzi ${ }^{1}$ \\ ${ }^{1}$ Food Science and Human Nutrition Research Unit, Medical Physiopatology, Food Science and Endocrinology \\ Section, Department of Experimental Medicine, Sapienza University of Rome, Rome, Italy \\ ${ }^{2}$ Unit of Endocrinology, Department of Movement, Human and Health Sciences, Foroltalico University of Rome, \\ Rome, Italy \\ Email: "lorenzomaria.donini@uniroma1.it
}

Received 6 August 2014; revised 3 September 2014; accepted 18 September 2014

Copyright (C) 2014 by authors and Scientific Research Publishing Inc.

This work is licensed under the Creative Commons Attribution International License (CC BY).

http://creativecommons.org/licenses/by/4.0/

(c) (i) Open Access

\begin{abstract}
Obesity and sarcopenia combination, appropriately defined as sarcopenic obesity (SO), due to disproportionally reduced/low lean body mass compared to excess fat mass, may lead to disability. Aims: The aim of our study was to investigate the relationship among sarcopenic obesity, physical performance, disability, and quality of life in a rehabilitation setting. Methods: Participants were recruited among obese patients (BMI $>30 \mathrm{~kg} / \mathrm{m}^{2}$ ) admitted to the rehabilitation facility at the Department of Experimental Medicine, Medical Physiopatology, Food Science and Endocrinology Section during a 1-year period. A multidimensional evaluation was performed through bioelectrical impedance analysis and anthropometry, handgrip strength test, Short Physical Performance Battery (SPPB), 6-minute walk test (6MWT) and blood chemistry parameters. Psychological status (SCL-90 questionnaire), quality of life, and comorbidity (Charlson comorbidity index score) were also evaluated. Obesity was diagnosed as increased fat mass by $35 \%$ in women and by $25 \%$ in men. Sarcopenia was defined if lean body mass (LBM) was $<90 \%$ of the subject's ideal LBM. Results: 79 patients (48 women and 31 men; mean age: $60.1 \pm 11.5$ years, and $58.6 \pm 10.8$ years, respectively) were enrolled. Results showed a high prevalence of SO (54.4\%) in our samples of obese subjects. Sarcopenia was present not only among older obese adults but also among younger obese subjects, and was related to reduced functional performance, to inflammatory status and to worse psychological status and quality of life.
\end{abstract}

\section{Keywords}

Sarcopenic Obesity, Disability

\footnotetext{
${ }^{*}$ Corresponding author.
}

How to cite this paper: Donini, L.M., Poggiogalle, E., Migliaccio, S., Pinto, A., Lubrano, C. and Lenzi, A. (2014) Sarcopenic Obesity: Correlation with Clinical, Functional, and Psychological Status in a Rehabilitation Setting. Food and Nutrition Sciences, 5, 2020-2031. http://dx.doi.org/10.4236/fns.2014.520213 


\section{Introduction}

Sarcopenic obesity is a critical public health related to two important phenomena: the rising prevalence of obesity in western and developing countries, and the increase of lifespan [1]-[4]. Changes in body composition, occurring in ageing as well as in obesity, represent the common soil where sarcopenic obesity develops [4].

A wealth of studies have focused on the reduction of lean body mass (LBM) in the elderly, that is simultaneously accompanied by the increase of the fat mass percentage, even when body weight and body mass index (BMI) remain stable [5]-[10]. The above mentioned changes in body composition associated with the ageing process have been demonstrated to occur also in obesity [5]-[10] (Figure 1). An imbalance between lean mass, excess body fat and total body size may appear earlier in obese adults [5] [6], because of the disproportion between the even conserved lean mass when compared to fat mass, exceeding body weight that lean mass could support.

Likewise, both sarcopenia and obesity are linked to functional impairment [11] [12]. Moreover, the associations between obesity per se and poor physical performance, as well as long-term consequences of sarcopenia on physical performance are potentially more severe in obese elder individuals [13]-[15].

Diagnostic criteria for sarcopenic obesity are not universally established, and different definitions are currently available. Hence, sarcopenic obesity prevalence ranges from $2.75 \%$ to over $20 \%$, depending on the criteria used for the diagnosis and methods of body composition assessment [6] [16]. Moreover the pathogenetic and functional role of sarcopenia or "relative" sarcopenia in obese elderly subjects as well as in younger adults, remains to be better clarified.

The aim of our study was to investigate the relationship among sarcopenic obesity, physical performance, disability, and quality of life.

\section{Methods}

\subsection{Subjects}

Participants were recruited among all obese patients admitted to the rehabilitation facility at the Department of Experimental Medicine—-Medical Physiopatology, Food Science and Endocrinology Section—from January to

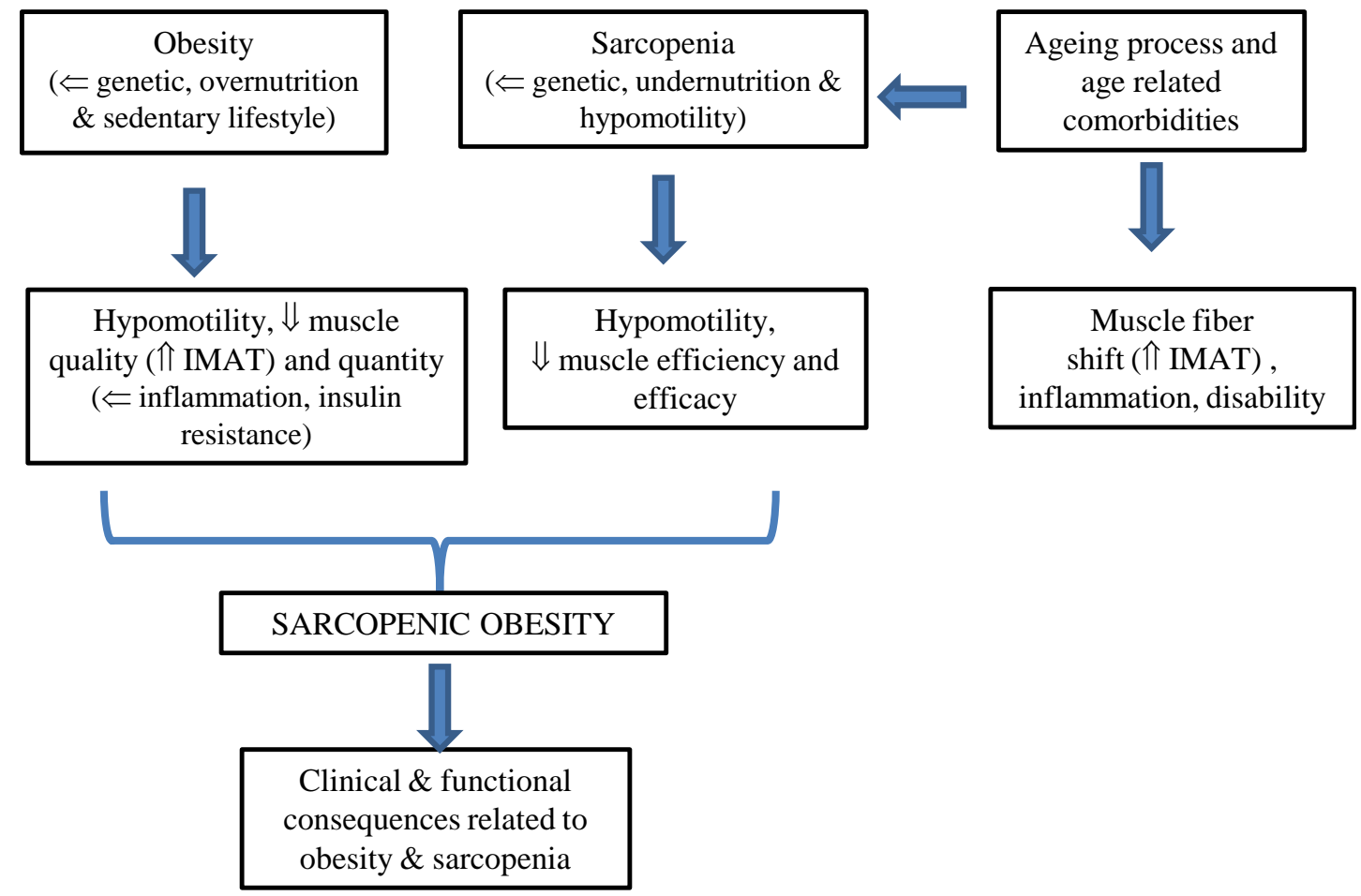

Figure 1. Sarcopenic obesity pathogenesis. Legend: IMAT = intramuscular adipose tissue. 
December 2011. Inclusion criteria were: age $\geq 18$ years; fat mass $>25 \%$ in men and $>35 \%$ in women; exclusion criteria were: sarcopenia associated to cancer cachexia or neurological diseases (CNS diseases), any malignant disease during the last five years, inflammatory myopathies, corticosteroids for systemic use, growth hormone replacement therapy, testosterone replacement therapy in males, sex hormone replacement therapy in females, bedridden subjects. All the evaluations (body composition, functional, health, disability and quality of life assessments, laboratory data) were performed during the first week after admission by a physician skilled in clinical nutrition.

The study was performed after the approval of the local ethics committee. Oral and written informed consent was obtained from all the participants.

\subsection{Body Composition}

Anthropometric measurements were taken following standardized procedures in accordance with the "Anthropometric standardization reference manual" by Lohman et al. [17]. Body weight was measured to the nearest 0.1 kg through a standard column body scale (SECA, Hamburg, Germany). Body height was determined using a rigid stadiometer (SECA, Hamburg, Germany) to the nearest $0.1 \mathrm{~cm}$. Body mass index (BMI) was calculated as body weight in $\mathrm{kg} /(\text { body height in } \mathrm{m})^{2}$.

Body composition [fat mass (FM) and LBM] was estimated by bioelectrical impedance analysis (BIA). The BIA measurement was performed following a standardized protocol (BIB) on the right side of the body using 800-A and 50-kHz alternating sinusoidal current and a standard tetrapolar technique (BIA 101 Impedance Analyzer, AKERN, Florence, Italy). Body composition was assessed through the Lukaski equation validated in obese subjects [18].

Lean body mass index (LBMI) was calculated as LBM in $\mathrm{kg} /(\text { body height in } \mathrm{m})^{2}$.

\subsection{Diagnosis of Obesity and Sarcopenia}

Obesity was diagnosed as fat mass increased by $35 \%$ in women and by $25 \%$ in men [19] [20]. LBM was considered depleted if it was $<90 \%$ of subject's ideal LBM (iLBM). Taking into account that fat mass in normal weight subjects corresponds to $15 \%$ in men and $25 \%$ in women [19] [20], ideal LBM was calculated in $\mathrm{kg}$ as the sum of $85 \%$ of ideal body weight in men or $75 \%$ in women, plus $25 \%$ of excess weight, expressed as body weight exceeding a reference body weight corresponding to a BMI $>25 \mathrm{~kg} / \mathrm{m}^{2}$, considering that excess body weight includes not only fat mass but also a certain amount of muscle mass [21] [22].

\subsection{Physical Function Assessment}

The hand-grip strength test (HGST) using the JAMAR Hydraulic Hand Dynamometer (Lafayette Instrument Company, USA), the Short Physical Performance Battery (SPPB), and the 6-minute walk test (6MWT) were performed in order to assess physical function and mobility [23]-[25]. Cut off values for HGST were $<20 \mathrm{~kg}$ for women and $<30 \mathrm{~kg}$ for men [26], while physical performance was considered impaired when total SPPB score was $\leq 8$ [26]. The 6-minute walked distance (6MWD) was compared to the predicted total distance walked during six minutes, computed using reference equations for the Italian population by Chetta et al. [27].

\subsection{Health, Disability and Quality of Life Assessment}

The collection of medical history and physical examination, as well as questionnaire administration, were performed on admission. Comorbid conditions were assessed using the Charlson comorbidity index score [28]. Disability was evaluated using the test for obesity-related disabilities (TSD-OC test) by the Italian Society of Obesity [29]. Quality of life was assessed by the administration of the Short-Form Health Survey questionnaire (SF-36 questionnaire) [30], and anxiety and depression were measured using the Symptom Checklist-90 (SCL90 questionnaire) [31].

\subsection{Laboratory Data}

Blood samples were obtained from the antecubital vein; blood sampling was performed in the early morning after an overnight fasting. 
Albumin, prealbumin, transferrin, hemoglobin, blood cell count and lymphocyte count, high-sensitivity C-reactive protein (HS-CRP), and cholinesterase levels were tested using commercial kits supplied by ABX Italy (Rome).

\subsection{Statistical Analysis}

After the verification of the normal distribution of the continuous variables, parametric tests were used to evaluate the difference between sarcopenic obese and nonsarcopenic obese subjects. The independent t-test was performed to describe differences between means of the groups. A linear regression analysis was performed to verify the association between body composition parameters (FM, LBM, real LBM/ideal LBM ratio) and clinical, functional and biochemical parameters.

Differences were considered to be statistically significant for $\mathrm{p}<0.05$. Statistical analysis was performed using SPSS 10.0 statistical software (SPSS Inc Wacker Drive, Chicago, IL, USA).

\section{Results}

A total of 79 subjects—48 women and 31 men—were enrolled in the study. They represent all the subjects admitted to the rehabilitation unit during the observation period. Mean age was: $60.1 \pm 11.5$ years for women and $58.6 \pm 10.8$ years for men respectively. Age and BMI were not statistically significantly different between genders, whereas statistically significant differences were found in terms of body composition (fat mass, LBM, LBMI and real LBM/ideal LBM ratio) as well as functional performance (handgrip strength test and SPPB score) and biochemical parameters (hemoglobin, lymphocytes, transferrin, albumin, prealbumin, cholinesterase levels), globally showing decreased values in females. No difference was observed with respect to depression, anxiety, quality of life, TSD-OC test score, HS-CRP, 6MWD, 6MWD/predicted-6MWD, and clinical status expressed by the Charlson comorbidity index (Table 1).

According with the definition used for diagnosing sarcopenia (real LBM/ideal LBM ratio < 0.9), 43 (54.4\%) obese subjects were sarcopenic. Sarcopenic obese subjects were older (mean age: $60.9 \pm 10.3$ versus $57.8 \pm 11.0$ years; $\mathrm{p}<0.05$ ); in particular, in our study population prevalence of sarcopenia was $52.9 \%$ in subjects aged less than or equal to 65 years and $57.1 \%$ in subjects older than 65 years $(\mathrm{p}=0.314)$. Sarcopenic obese subjects had a reduced BMI $\left(44.2 \pm 6.2\right.$ versus $\left.46.9 \pm 8.3 \mathrm{~kg} / \mathrm{m}^{2} ; \mathrm{p}<0.05\right)$ due to the observed significant reduction of LBM (both total LBM-expressed in kg - and LBMI were significantly reduced) and, to a lesser extent, to a decreased amount of FM, when compared to their non-sarcopenic obese counterparts. Functional parameters (in particular hand grip strength and disability) were significantly reduced $(\mathrm{p}<0.05)$, while a trend toward decreased values, but no statistically significant difference, was found for 6MWD and SPPB score, confirming a lower functional capacity in sarcopenic obese subjects. Concerning clinical and laboratory parameters, significant differences were found just for hemoglobin and albumin, that were significantly reduced in sarcopenic obese subjects, while their HS-CRP levels were significantly higher than nonsarcopenic obese subjects $(\mathrm{p}<0.05)$. Quality of life (in particular physical health-related quality of life), expressed by the SF-36 questionnaire score, was significantly impaired, while depression, anxiety and SCL-90 questionnaire total score were significantly increased in sarcopenic obese subjects when compared to nonsarcopenic obese subjects (Table 2).

In Table 3 the correlation between real LBM/ideal LBM ratio and clinical, functional and laboratory parameters is described. A negative significant correlation was found with age, disability (TSD-OC test), HS-CRP and depression score at the SCL-90 questionnaire. A positive significant correlation was found between real LBM/ideal LBM ratio and BMI, handgrip strength, functional performance (SPPB score), hemoglobin, serum transport proteins (albumin, transferrin, and prealbumin), and quality of life (physical health-related quality of life and SF-36 questionnaire total score).

Table 4 shows the correlation between different parameters defining fat mass (FM as percentage of body weight, total FM in kg, and FMI) and lean body mass (LBM as percentage of body weight, total LBM in kg, and FMI) and functional parameters. LBM was positively correlated with handgrip strength and SPPB score, and negatively correlated with disability (TSD-OC test score) $(\mathrm{p}<0.05)$.

On the contrary, FM was negatively correlated with SPPB score, and positively correlated with disability (TSD-OC test score) $(\mathrm{p}<0.05)$. No correlation was found between FM and handgrip strength, and between FM and LBM with the distance walked at the 6MWT. 
Table 1. Demographic characteristics and anthropometric parameters of the study population.

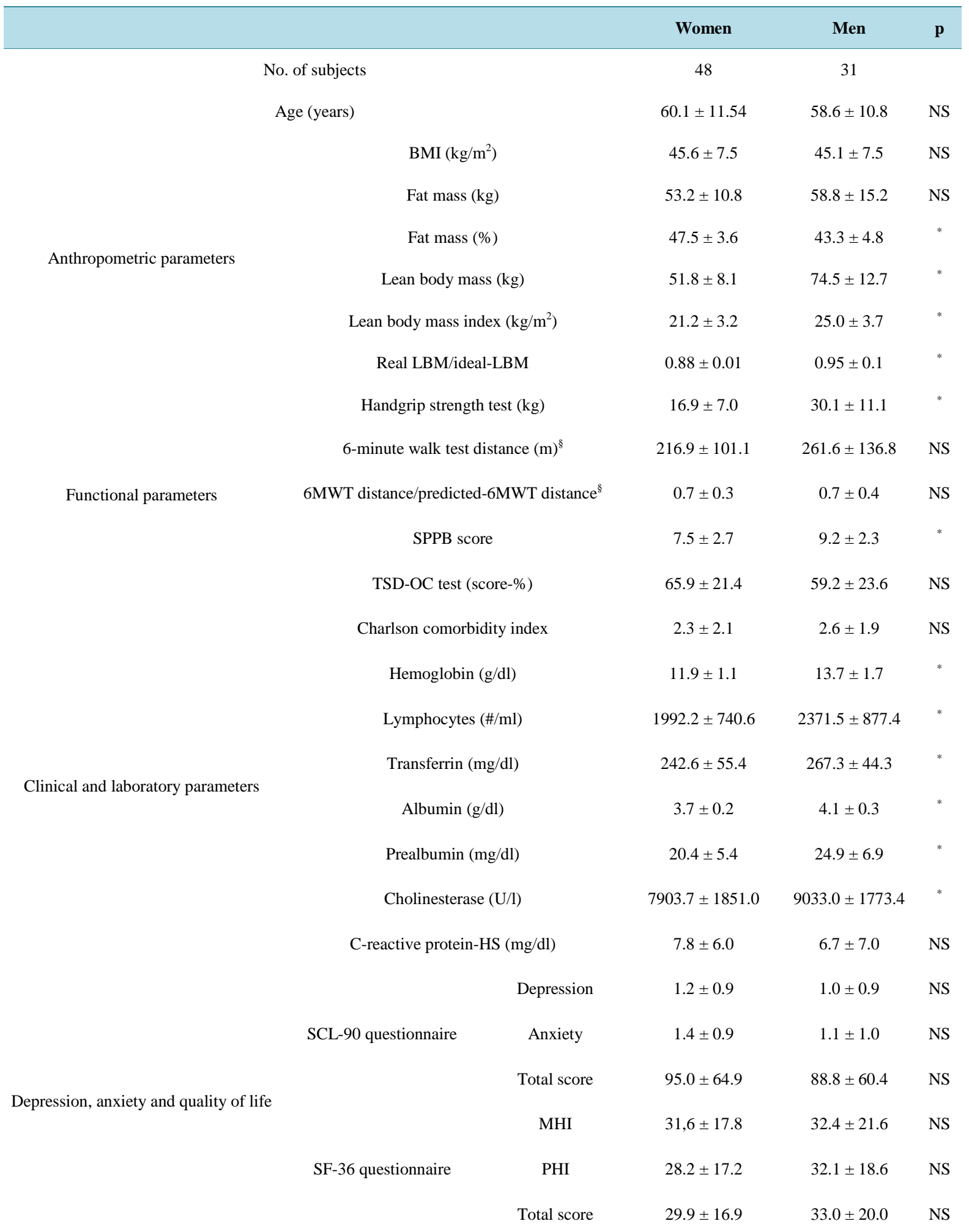

Legend: BMI: body mass index; LBM: fat free mass; 6MWT: six minutes walk test; SPPB: short physical performance battery; TSD-OC: Italian society of obesity test for obesity-related disabilities; SF-36: short-form health survey questionnaire; SCL-90: symptom checklist-90. ${ }^{\S} 18 / 48$ women (37.5\%) and 12/31 men (38.7\%) did not complete the 6MWT due to cardiovascular, respiratory or osteoarticular problems. ${ }^{*} \mathrm{p}<0.05$; NS: not statistically significant. 
Table 2. Clinical, functional and laboratory parameters and subjects’ characteristics related to real LBM-real/ideal LBM ratio.

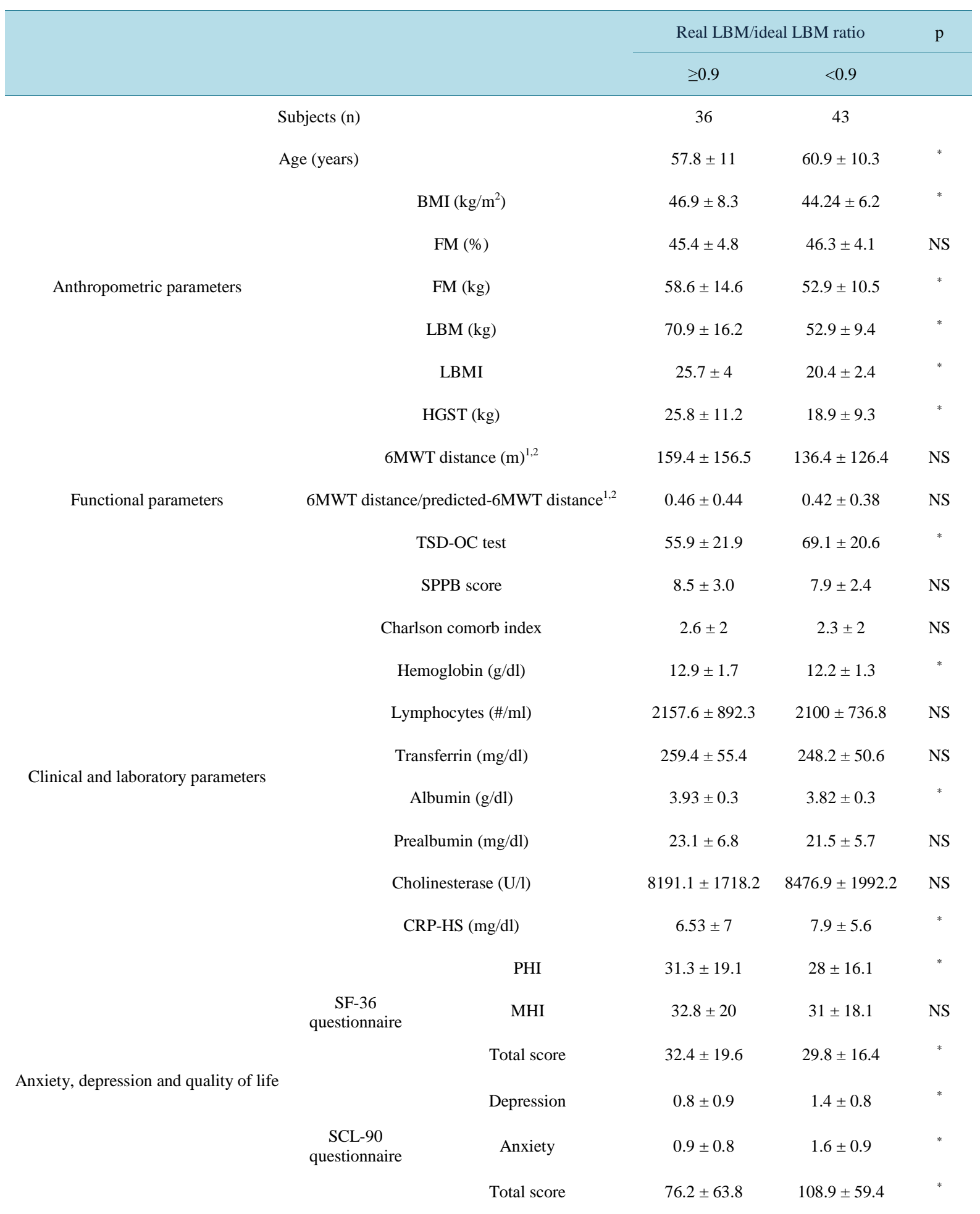

Legend: BMI: body mass index; FM: fat mass; LBM: fat free mass; LBMI: fat free mass index; HGST: handgrip strength; 6MWT: six minutes walk test; SPPB: short physical performance battery; TSD-OC: Italian society of obesity test for obesity-related disabilities; SF-36: short-form health survey questionnaire; SCL-90: symptom checklist-90; CRP: high sensitivity C-reactive protein. Notes: ${ }^{1} 15 / 43$ non-sarcopenic (34.9\%) and $15 / 36$ sarcopenic $(41.7 \%)$ did not complete the $6 \mathrm{MWT} ;{ }^{2} 18 / 48$ women $(37.5 \%)$ and $12 / 31$ men $(38.7 \%)$ did not complete the $6 \mathrm{MWT}$ due to cardiovascular, respiratory or osteoarticular problems. ${ }^{*} \mathrm{p}<0.05$; NS: not statistically significant. 
Table 3. Correlation between real LBM/ideal LBM ratio and clinical, functional and laboratory parameters.

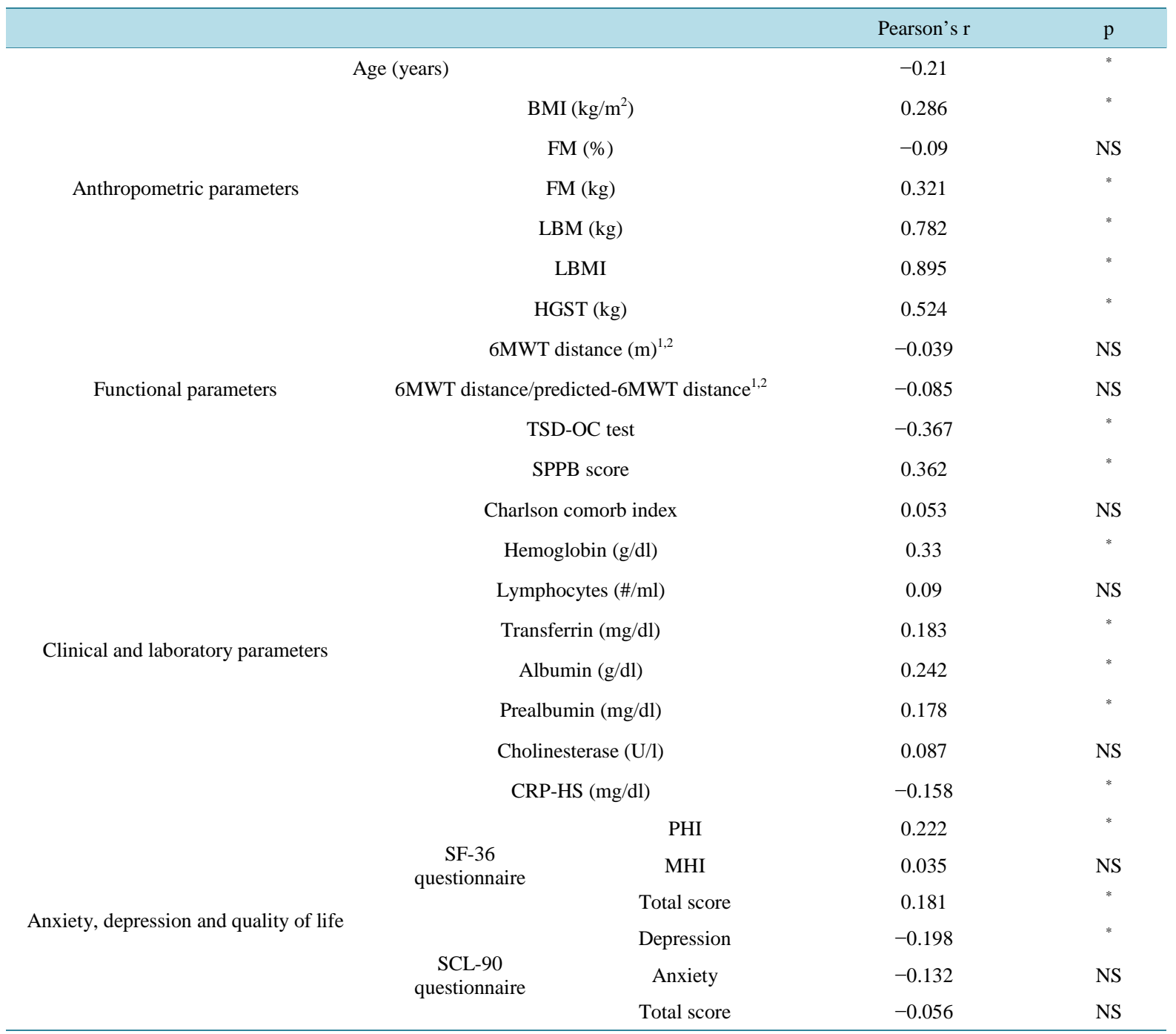

Legend: BMI: body mass index; FM: fat mass; LBM: fat free mass; LBMI: fat free mass index; HGST: handgrip strength; 6MWT: six minutes walk test; SPPB: short physical performance battery; TSD-OC: Italian society of obesity test for obesity-related disabilities; SF-36: short-form health survey questionnaire; SCL-90: symptom checklist-90; CRP: high sensitivity C-reactive protein. Notes: ${ }^{1} 15 / 43$ non-sarcopenic (34.9\%) and 15/36 sarcopenic $(41.7 \%)$ did not complete the 6MWT; ${ }^{2} 18 / 48$ women (37.5\%) and 12/31 men (38.7\%) did not complete the 6MWT due to cardiovascular, respiratory or osteoarticular problems. ${ }^{*} \mathrm{p}<0.05$; NS: not statistically significant.

Table 4. Correlation between body composition (fat mass and free-mass) and functional parameters.

\begin{tabular}{cccccccc}
\hline & \multicolumn{3}{c}{ Fat mass } & \multicolumn{3}{c}{ Fat free mass } \\
\hline & FM (\%) & FM (kg) & FMI & LBM (\%) & LBM (kg) & LBMI \\
\hline HGST (kg) & -0.137 & -0.155 & -0.106 & $0.357^{*}$ & $0.469^{*}$ & $0.492^{*}$ \\
6MWT (m) & -0.169 & -0.079 & -0.107 & 0.072 & 0.084 & 0.059 \\
6MWT distance/predicted-6MWT distance ${ }^{1,2}$ & -0.15 & -0.115 & -0.104 & 0.037 & 0.021 & 0.115 \\
TSD-OC test & $0.243^{*}$ & $0.234^{*}$ & $0.266^{*}$ & $-0.268^{*}$ & $-0.253^{*}$ & $-0.296^{*}$ \\
SPPB score & $-0.449^{*}$ & $-0.172^{*}$ & $-0.394^{*}$ & $0.326^{*}$ & $0.233^{*}$ & $0.331^{*}$ \\
\hline
\end{tabular}

Legend: FM: fat mass; LBM: fat free mass; LBMI: fat free mass index; HGST: handgrip strength; 6MWT: six minutes walk test; SPPB: short physical performance battery; TSD-OC: Italian society of obesity test for obesity-related disabilities. Notes: ${ }^{1} 15 / 43$ non-sarcopenic (34.9\%) and $15 / 36$ sarcopenic $(41.7 \%)$ did not complete the $6 \mathrm{MWT}{ }^{2} 18 / 48$ women $(37.5 \%)$ and $12 / 31$ men $(38.7 \%)$ did not complete the $6 \mathrm{MWT}$ due to cardiovascular, respiratory or osteoarticular problems. ${ }^{*} \mathrm{p}<0.05$. 


\section{Discussion}

In the present study we found a high prevalence of sarcopenia (defined as real LBM/ideal LBM ratio) in a sample of obese subjects. Sarcopenia was present not only among older obese adults, but also in pre-geriatric obese subjects. Furthermore, SO was related to reduced functional performance, to inflammatory status, to a worse psychological status as well as to reduced quality of life.

Diagnosis of sarcopenic obesity is a challenging clinical issue. Existing prevalence data are very variable and inconsistent because of the use of different methods for diagnosis (see a recent systematic review on this topic [32]), and most of the extant studies involved only older adults [5] [16] [26] [33]. In our study we evaluated sarcopenia using the ratio between LBM estimated by BIA and the ideal LBM. In our opinion and according with our data, the ratio between real LBM and ideal LBM may represent an additional indicator of sarcopenia, and a ratio less than 0.9 may be considered indicative of sarcopenia. Anyway, these data need to be confirmed by future studies. In accordance with the extant literature about body composition in adult and healthy humans, 85\% in men and $75 \%$ in women, respectively, of the normal body weight consists of lean mass. In addition, as suggested by a number of studies about body composition and obesity, in obese subjects excess body weight is not only made of fat mass, but it includes a certain amount of muscle mass, that seems to correspond to $25 \%$ of excess body weight [19] [22] [23] [34]-[36]. The augmentation of LBM paralleling the increase of body weight would be a protective mechanism allowing the obese individual to sustain the gained fat mass, avoiding, at least in the early phases of the process, the onset of metabolic and functional impairment as fat mass increases. In fact, on the basis of the above mentioned evidence and our data, we hypothesize that a "relative" sarcopenia, before the development of an "absolute" sarcopenia, is predictive of disability in obese subjects. This remark is consistent with observations showing that abnormalities in body composition, affecting lean mass, occur in obese subjects even prior to the old age, overall due to inflammation, dieting and weight cycling phenomena, and engagement in sedentary activities [36]-[39]. Few studies showed that weight regain after weight loss may be at the basis of changes in body composition leading, at the end of the cycle, paradoxically to an increased amount of body fat [39] [40]. The combination of these mechanisms, the former protective, the latter detrimental towards lean body mass, may account for discrepancies from previous studies; the use of the real LBM/ideal LBM ratio may be effective in reducing and overcoming these differences.

In our study, prevalence of sarcopenia in obese subjects was higher than sarcopenic obesity prevalence reported from available prevalence studies [5] [16] [33]. This finding could be potentially explained by the setting of recruitment in our study: all the subjects were enrolled in a rehabilitation unit devoted to the nutritional and metabolic rehabilitation of subjects with obesity or eating disorders. Clinical status and disability in our study population were consistent with a more deteriorated clinical condition and functional performance, even if many obese participants were younger than 65 years. Another potential explanation may be related to the normative value that we used (real LBM/ideal LBM ratio < 0.9). This cut-off needs to be verified in a larger sample of obese subjects. However, the correlation found in our study between real LBM/ideal LBM ratio and functional parameters let us argue that this value is sufficiently correct.

In the present study disability was assessed through the TSD-OC test, a recently validated tool specifically designed to assess disability level in obese subjects [29]. The TSD-OC test is composed of 36 items divided into seven sections (pain, stiffness, activities of daily living and indoor mobility, housework, outdoor activities, occupational activities and social life). In the validation study the TSD-OC test was found to be significantly correlated to functional assessment (6MWD and handgrip strength) and quality of life parameters (SF-36 questionnaire). Our results highlight that the presence of sarcopenia in obese subjects was associated with a worse TSD-OC test score; this finding is consistent with data provided by a recent study by Baumgartner, pointing out the coexistence of low physical capacity and sarcopenic obesity [10]. These observations support the synergic pathological action exerted by obesity and sarcopenia; however, it is still matter of debate which of the two components (increased FM or reduced LBM) in sarcopenic obesity is better correlated with disability. Different studies concluded that excess body fat was a stronger contributor to physical function impairment than sarcopenia [41] [42]. Davison and coll. did not demonstrate any association between sarcopenia and functional limitations, instead mobility impairment was related to percentage of body fat and BMI [13]. Similarly, Cawthon et al. found that adipose tissue and performance status were more closely related to disability than lean body mass [43].

In our study, decreased LBM and increased FM were independently and statistically significantly associated with different functional parameters suggesting that both sarcopenia and obesity account for disability. The ef- 
fect of fat mass on physical function is under debate and some Authors consider that it may be as great as that of muscle mass, accounting for the U-shaped relationship between BMI and physical limitations [44]. In our study the correlation was stronger for LBM and especially for the real LBM/ideal LBM ratio. We stress the need for a more precise assessment of body composition, taking into account the role of sarcopenia as a pivotal determinant of disability in obese subjects. Few studies tried to consider the effect of both fat mass and lean body mass on disability. In a recent paper, Authors hypothesized that disability caused by sarcopenia and sarcopenic obesity was related to the amount of adiposity or body weight bearing on a unit of muscle mass (total body fat to lower limb muscle mass ratio) [45]. Our finding of increased disability in obese subjects with sarcopenia confirms that the sarcopenia, in the context of obesity, may be better defined as a "relative" sarcopenia: despite the appearance that muscle mass is conserved, it probably is not enough in proportion to the total body mass to prevent the onset of functional impairment and disability. Moreover, fat infiltration of muscles, named "myosteatosis" [46], could also be responsible for deteriorated muscle strength in obese individuals. Changes in body composition and musche strength related to the ageing process and in particular to changes in body composition are associated with functional decline [47].

One of the more significant findings emerging from our study is that sarcopenia was found also in relatively young obese subjects. Papers reporting analogous data are scarce [48]. In line with the hypothesis that obesity produces low-grade inflammation and hormonal changes affecting muscle function and metabolism, obese young people could have similar changes and meet the criteria for the diagnose of sarcopenic obesity [48]. Moreover pronounced and prolonged inactivity together with repeated unbalanced diets leading to rapid weight loss may deeply affect lean body mass.

One limitation to our study was the use of BIA in order to assess muscle mass, that is less precise than DXA or other imaging methods like magnetic resonance or CT-scan. On the other hand, as in most of the clinical facilities, in our rehabilitation unit DXA scanner machine held a maximum body weight of $130 \mathrm{~kg}$, and it would have made impossible the DXA analysis of the severely obese participants, who are usually the most represented obese individuals in our metabolic-nutritional-psychological rehabilitation unit. However, in line with EWGSOP recommendations, BIA is an alternative to expensive imaging techniques, when performed under standard conditions [26] [32] [49]-[51]. Moreover considering 35\% and 25\%, respectively for males and females, as the cut off point of FM to define obesity does not take into account age related changes. However the data in the literature, similarly to what happens for BMI, do not allow a more precise definition of obesity based on FM and age classes. At last the sample size is limited and do not allow a more in depth evaluation for confounders through complex models as it would be appropriate in a cross-sectional study.

Finally, we are aware that an exhaustive definition of sarcopenia needs a thorough evaluation considering body composition, muscle strength and physical performance. Our study may be a first step to better define LBM depletion in sarcopenic obesity.

\section{Conclusions}

To conclude, sarcopenia (defined through real LBM/ideal LBM ratio) in obese subjects seems to be correlated to disability, reduced functional performance, worse psychological status and reduced quality of life. Further research should be prompted:

-in order to confirm the validity of the proposed definition of sarcopenia adapted to obese subjects, focusing on muscle quality and composition;

-to define specific nutritional, psychological, and functional rehabilitation programs for sarcopenic obese subjects, aimed at modifying body composition (increasing LBM while decreasing FM) and improving functional parameters and quality of life [52].

\section{Disclosure Statement}

None of the authors had any conflicts of interest nor financial interest. All authors attest and affirm that the material within has not been and will not be submitted for publication elsewhere.

\section{References}

[1] Baumgartner, R.N. (2000) Body Composition in Healthy Aging. Annals of the New York Academy of Sciences, 904, 437-448. http://dx.doi.org/10.1111/j.1749-6632.2000.tb06498.x 
[2] Flegal, K.M., Carroll, M.D., Ogden, C.L. and Johnson, C.L. (2002) Prevalence and Trends in Obesity among US Adults, 1999-2000. JAMA, 288, 1723-1727. http://dx.doi.org/10.1001/jama.288.14.1723

[3] Villareal, D.T, et al. (2009) Obesity in Older Adults-A Growing Problem. Nutrition and Health: Handbook of Clinical Nutrition and Aging. 2nd Edition, Humana Press, New York, 263-277.

[4] Roubenoff, R. (2004) Sarcopenic Obesity: The Confluence of Two Epidemics. Obesity Research, 12, 887-888. http://dx.doi.org/10.1038/oby.2004.107

[5] Stenholm, S., Harris, T.B., Rantanen, T., Visser, M., Kritchevsky, S.B. and Ferrucci, L. (2008) Sarcopenic Obesity: Definition, Cause and Consequences. Current Opinion in Clinical Nutrition and Metabolic Care, 11, 693-700. http://dx.doi.org/10.1097/MCO.0b013e328312c37d

[6] Waters, D.L. and Baumgartner, R.N. (2011) Sarcopenia and Obesity. Clinics in Geriatric Medicine, 27, 401-421. http://dx.doi.org/10.1016/j.cger.2011.03.007

[7] Zamboni, M., Mazzali, G., Fantin, F., Rossi, A. and Di Francesco, V. (2008) Sarcopenic Obesity: A New Category of Obesity in the Elderly. Nutrition, Metabolism Cardiovascular Diseases, 18, 388-395. http://dx.doi.org/10.1016/j.numecd.2007.10.002

[8] Kim, T.N., Yang, S.J., Yoo, H.J., Lim, K.I., Kang, H.J., Song, W., Seo, J.A., Kim, S.G., Kim, N.H., Baik, S.H., Choi, D.S. and Choi, K.M. (2009) Prevalence of Sarcopenia and Sarcopenic Obesity in Korean Adults: The Korean Sarcopenic Obesity Study. International Journal of Obesity, 33, 885-892. http://dx.doi.org/10.1038/ijo.2009.130

[9] Dominguez, L.J. and Barbagallo, M. (2007) The Cardiometabolic Syndrome and Sarcopenic Obesity in Older Persons. Journal of the Cardiometabolic Syndrome, 2, 183-189. http://dx.doi.org/10.1111/j.1559-4564.2007.06673.x

[10] Baumgartner, R.N., Wayne, S.J., Waters, D.L., Janssen, I., Gallagher, D. and Morley, J.E. (2004) Sarcopenic Obesity Predicts Instrumental Activities of Daily Living Disability in the Elderly. Obesity Research, 12, 1995-2004. http://dx.doi.org/10.1038/oby.2004.250

[11] Zamboni, M., Mazzali, G., Zoico, E., Harris, T.B., Meigs, J.B., Di Francesco, V., Fantin, F., Bissoli, L. and Bosello, O. (2005) Health Consequences of Obesity in the Elderly: A Review of Four Unresolved Questions. International Journal of Obesity, 29, 1011-1029. http://dx.doi.org/10.1038/sj.ijo.0803005

[12] Ritz, P. (2009) Obesity in the Elderly: Should We Be Using New Diagnostic Criteria? Journal of Nutrition, Health and Aging, 13, 168-169. http://dx.doi.org/10.1007/s12603-009-0052-7

[13] Davison, K.K., Ford, E.S., Cogswell, M.E. and Dietz, W.H. (2002) Percentage of Body Fat and Body Mass Index Are Associated with Mobility Limitation in People Aged 70 and Older from NHANES III. Journal of the American Geriatrics Society, 50, 1802-1809. http://dx.doi.org/10.1046/j.1532-5415.2002.50508.x

[14] Zoico, E., Di Francesco, V., Guralnik, J.M., Mazzali, G., Bortolani, A., Guariento, S., Sergi, G., Bosello, O. and Zamboni, M. (2004) Physical Disability and Muscular Strength in Relation to Obesity and Different Body Composition Indexes in a Sample of Healthy Elderly Women. International Journal of Obesity, 28, 234-241.

[15] Baumgartner, R.N., Wayne, S.J., Waters, D.L., Janssen, I., Gallagher, D. and Morley, J.E. (2004) Sarcopenic Obesity Predicts Instrumental Activities of Daily Living Disability in the Elderly. Obesity Research, 12, 1995-2004. http://dx.doi.org/10.1038/oby.2004.250

[16] Prado, C.M., Wells, J.C., Smith, S.R., Stephan, B.C. and Siervo, M. (2012) Sarcopenic Obesity: A Critical Appraisal of the Current Evidence. Clinical Nutrition, 31, 583-601. http://dx.doi.org/10.1016/j.clnu.2012.06.010

[17] Lohman, T.G., Roche, A.F. and Martorell, R. (1992) Manuale di riferimento per la standardizzazione antropometrica. EDRA, Milano.

[18] Lukaski, H.C., Bolonchuk, W.W., Hall, C.B. and Siders, W.A. (1986) Validation of Tetrapolar Bioelectrical Impedance Method to Assess Human Body Composition. Journal of Applied Physiology, 60, 1327-1332.

[19] Deurenberg, P., Yap, M. and van Staveren, W.A. (1998) Body Mass Index and Percent Body Fat: A Meta-Analysis among Different Ethnic Groups. International Journal of Obesity, 22, 1164-1171. http://dx.doi.org/10.1038/sj.ijo.0800741

[20] Report of a WHO Expert Committee (1995) Physical Status: The Use and Interpretation of Anthropometry. WHO Technical Report Series 854, Geneva.

[21] Joosen, A.M., Bakker, A.H. and Westerterp, K.R. (2005) Metabolic Efficiency and Energy Expenditure during ShortTerm Overfeeding. Physiology and Behaviour, 85, 593-597. http://dx.doi.org/10.1016/j.physbeh.2005.06.006

[22] Norgan, N.G. and Durnin, J.V. (1980) The Effect of 6 Weeks of Overfeeding on the Body Weight, Body Composition, and Energy Metabolism of Young Men. American Journal of Clinical Nutrition, 33, 978-988.

[23] Günther, C.M., Bürger, A., Rickert, M., Crispin, A. and Schulz, C.U. (2008) Grip Strength in Healthy Caucasian Adults: Reference Values. Journal of Hand Surgery, 33, 558-565. http://dx.doi.org/10.1016/j.jhsa.2008.01.008

[24] Guralnik, J.M., Simonsick, E.M., Ferrucci, L., Glynn, R.J., Berkman, L.F., Blazer, D.G., Scherr, P.A. and Wallace, R.B. 
(1994) A Short Physical Performance Battery Assessing Lower Extremity Function: Association with Self-Reported Disability and Prediction of Mortality and Nursing Home Admission. Journal of Gerontology, 49, M85-M94. http://dx.doi.org/10.1093/geronj/49.2.M85

[25] (2002) ATS Statement: Guidelines for the Six-Minute Walk Test. American Journal of Respiratory and Critical Care Medicine, 166, 111-117. http://dx.doi.org/10.1164/ajrccm.166.1.at1102

[26] Cruz-Jentoft, A.J., Baeyens, J.P., Bauer, J.M., Boirie, Y., Cederholm, T., Landi, F., Martin, F.C., Michel, J.P., Rolland, Y., Schneider, S.M., Topinková, E., Vandewoude, M. and Zamboni, M. (2010) Sarcopenia: European Consensus on Definition and Diagnosis: Report of the European Working Group on Sarcopenia in Older People. Age and Ageing, 39, 412-423. http://dx.doi.org/10.1093/ageing/afq034

[27] Chetta, A., Zanini, A., Pisi, G., Aiello, M., Tzani, P., Neri, M. and Olivieri, D. (2006) Reference Values for the 6-min Walk Test in Healthy Subjects 20 - 50 Years Old. Respiratory Medicine, 100, 1573-1578. http://dx.doi.org/10.1016/j.rmed.2006.01.001

[28] Charlson, M., Szatrowski, T.P., Peterson, J. and Gold, J. (1994) Validation of a Combined Comorbidity Index. Journal of Clinical Epidemiology, 47, 1245-1251. http://dx.doi.org/10.1016/0895-4356(94)90129-5

[29] Donini, L.M., Brunani, A., Sirtori, A., Savina, C., Tempera, S., Cuzzolaro, M., Spera, G., Cimolin, V., Precilios, H., Raggi, A. and Capodaglio, P. (2011) Assessing Disability in Morbidly Obese Individuals: The Italian Society of Obesity Test for Obesity-Related Disabilities. Disability and Rehabilitation, 33, 2509-2518. http://dx.doi.org/10.3109/09638288.2011.575529

[30] Apolone, G. and Mosconi, P. (1998) The Italian SF-36 Health Survey: Translation, Validation and Norming. Journal of Clinical Epidemiology, 51, 1025-1036. http://dx.doi.org/10.1016/S0895-4356(98)00094-8

[31] Derogatis, L.R. and Savitz, K.L. (2000) The SCL-90-R and the Brief Symptom Inventory (BSI) in Primary Care. In: Maruish, M.E., Ed., Handbook of Psychological Assessment in Primary Care Settings, Vol. 236, Lawrence Erlbaum Associates, Mahwah, 297-334.

[32] Donini, L.M., Poggiogalle, E., Migliaccio, S., Aversa, A. and Pinto, A. (2013) Body Composition in Sarcopenic Obesity: Systematic Review of the Literature. Mediterranean Journal of Nutrition and Metabolism, 6, 191-198.

[33] Janssen, I. (2011) The Epidemiology of Sarcopenia. Clinics in Geriatric Medicine, 27, 355-363. http://dx.doi.org/10.1016/j.cger.2011.03.004

[34] Passmore, R., Meiklejohn, A.P., Dewar, A.D. and Thow, R.K. (1955) An Analysis of the Gain in Weight of Overfed Thin Young Men. British Journal of Nutrition, 9, 27-37. http://dx.doi.org/10.1079/BJN19550007

[35] Deurenberg, P., Yap, M.D., Wang, J., Lin, F.P. and Schmidt, G. (1999) The Impact of Body Build on the Relationship between Body Mass Index and Percent Body Fat. International Journal of Obesity, 23, 537-542. http://dx.doi.org/10.1038/sj.ijo.0800868

[36] Gallagher, D. and DeLegge, M. (2011) Body Composition (Sarcopenia) in Obese Patients: Implications for Care in the Intensive Care Unit. Journal of Parenteral and Enteral Nutrition, 35, 21S-28S. http://dx.doi.org/10.1177/0148607111413773

[37] Roth, S.M., Metter, E.J., Ling, S. and Ferrucci, L. (2006) Inflammatory Factors in Age-Related Muscle Wasting. Current Opinion in Rheumatology, 18, 625-630. http://dx.doi.org/10.1097/01.bor.0000245722.10136.6d

[38] Visser, M., Pahor, M., Taaffe, D.R., Goodpaster, B.H., Simonsick, E.M., Newman, A.B., Nevitt, M. and Harris, T.B. (2002) Relationship of Interleukin-6 and Tumor Necrosis Factor-Alpha with Muscle Mass and Muscle Strength in Elderly Men and Women: The Health ABC Study. Journals of Gerontology Series A: Biological Sciences and Medical Sciences, 57, M326-M332. http://dx.doi.org/10.1093/gerona/57.5.M326

[39] Forbes, G.B. (1999) Longitudinal Changes in Adult Fat-Free Mass: Influence of Body Weight. American Journal of Clinical Nutrition, 70, 1025-1031.

[40] Lee, J.S., Visser, M., Tylavsky, F.A., Kritchevsky, S.B., Schwartz, A.V., Sahyoun, N., Harris, T.B. and Newman, A.B., For the Health ABC Study (2010) Weight Loss and Regain and Effects on Body Composition: The Health, Aging, and Body Composition Study. Journals of Gerontology Series A: Biological Sciences and Medical Sciences, 65, 78-83. http://dx.doi.org/10.1093/gerona/glp042

[41] Bouchard, D.R., Dionne, I.J. and Brochu, M. (2009) Sarcopenic/Obesity and Physical Capacity in Older Men and Women: Data from the Nutrition as a Determinant of Successful Aging (NuAge)—The Quebec Longitudinal Study. Obesity (Silver Spring), 17, 2082-2088. http://dx.doi.org/10.1038/oby.2009.109

[42] Jankowski, C.M., Gozansky, W.S., Van Pelt, R.E., Schenkman, M.L., Wolfe, P., Schwartz, R.S. and Kohrt, W.M. (2008) Relative Contributions of Adiposity and Muscularity to Physical Function in Community-Dwelling Older Adults. Obesity (Silver Spring), 16, 1039-1044. http://dx.doi.org/10.1038/oby.2007.84

[43] Cawthon, P.M., Fox, K.M., Gandra, S.R., Delmonico, M.J., Chiou, C.F., Anthony, M.S., Caserotti, P., Kritchevsky, S.B., Newman, A.B., Goodpaster, B.H., Satterfield, S., Cummings, S.R. and Harris, T.B., For the Health, Aging and 
Body Composition Study (2011) Clustering of Strength, Physical Function, Muscle, and Adiposity Characteristics and Risk of Disability in Older Adults. Journal of the American Geriatrics Society, 59, 781-787. http://dx.doi.org/10.1111/j.1532-5415.2011.03389.x

[44] Woo, J., Leung, J. and Kwok, T. (2007) BMI, Body Composition, and Physical Functioning in Older Adults. Obesity (Silver Spring), 15, 1886-1894.

[45] Auyeung, T.W., Lee, J.S., Leung, J., Kwok, T. and Woo, J. (2013) Adiposity to Muscle Ratio Predicts Incident Physical Limitation in a Cohort of 3,153 Older Adults-An Alternative Measurement of Sarcopenia and Sarcopenic Obesity. Age, 35, 1377-1385. http://dx.doi.org/10.1007/s11357-012-9423-9

[46] Miljkovic, I. and Zmuda, J.M. (2010) Epidemiology of Myosteatosis. Current Opinion in Clinical Nutrition and Metabolic Care, 13, 260-264. http://dx.doi.org/10.1097/MCO.0b013e328337d826

[47] Schaap, L.A., Koster, A. and Visser, M. (2013) Adiposity, Muscle Mass, and Muscle Strength in Relation to Functional Decline in Older Persons. Epidemiologic Reviews, 35, 51-65. http://dx.doi.org/10.1093/epirev/mxs006

[48] Gonzalez-Correa, C.H., Caicedo-Eraso, J.C. and Villada-Gomez, J.S. (2013) Bioelectrical Impedance Analysis for Sarcopenic Obesity Diagnosis in Young Female Subjects. Journal of Physics: Conference Series, 434, Article ID: 012061.

[49] Kyle, U.G., Genton, L., Karsegard, L., Slosman, D.O. and Pichard, C. (2001) Single Prediction Equation for Bioelectrical Impedance Analysis in Adults Aged 20 - 94 Years. Nutrition, 17, 248-253. http://dx.doi.org/10.1016/S0899-9007(00)00553-0

[50] Chien, M.Y., Huang, T.Y. and Wu, Y.T. (2008) Prevalence of Sarcopenia Estimated Using a Bioelectrical Impedance Analysis Prediction Equation in Community-Dwelling Elderly People in Taiwan. Journal of the American Geriatrics Society, 56, 1710-1715.

[51] Kyle, U.G., Bosaeu, I., De Lorenzo, A., Deurenberg, P., Elia, M., Gomez, J.M., Heitmann, B.L., Kent-Smith, L., Melchior, J.C., Pirlich, M., Scharfetter, H., Schols, A. and Pichard, C. (2004) Bioelectrical Impedance Analysis-Part II: Utilization in Clinical Practice. Clinical Nutrition, 23, 1430-1453. http://dx.doi.org/10.1016/j.clnu.2004.09.012

[52] Poggiogalle, E., Migliaccio, S., Lenzi, A. and Donini, L.M. (2014) Treatment of Body Composition Changes in Obese and Overweight Older Adults: Insight into the Phenotype of Sarcopenic Obesity. Endocrine. 
Scientific Research Publishing (SCIRP) is one of the largest Open Access journal publishers. It is currently publishing more than 200 open access, online, peer-reviewed journals covering a wide range of academic disciplines. SCIRP serves the worldwide academic communities and contributes to the progress and application of science with its publication.

Other selected journals from SCIRP are listed as below. Submit your manuscript to us via either submit@scirp.org or Online Submission Portal.
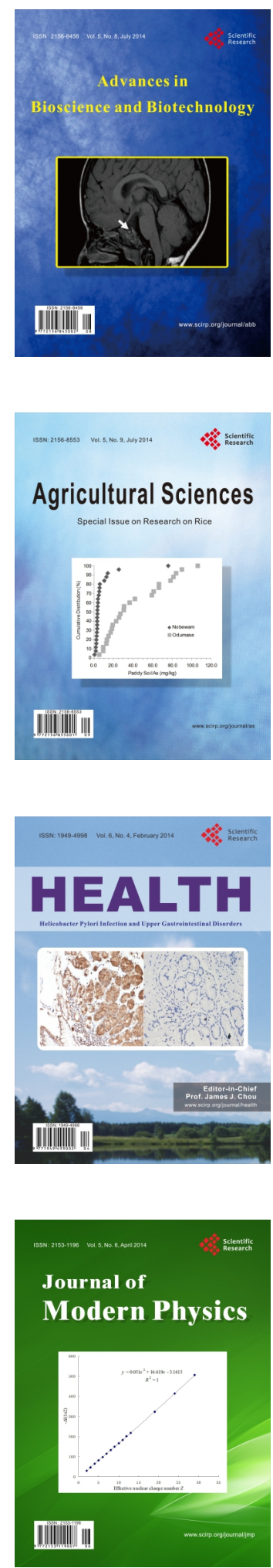
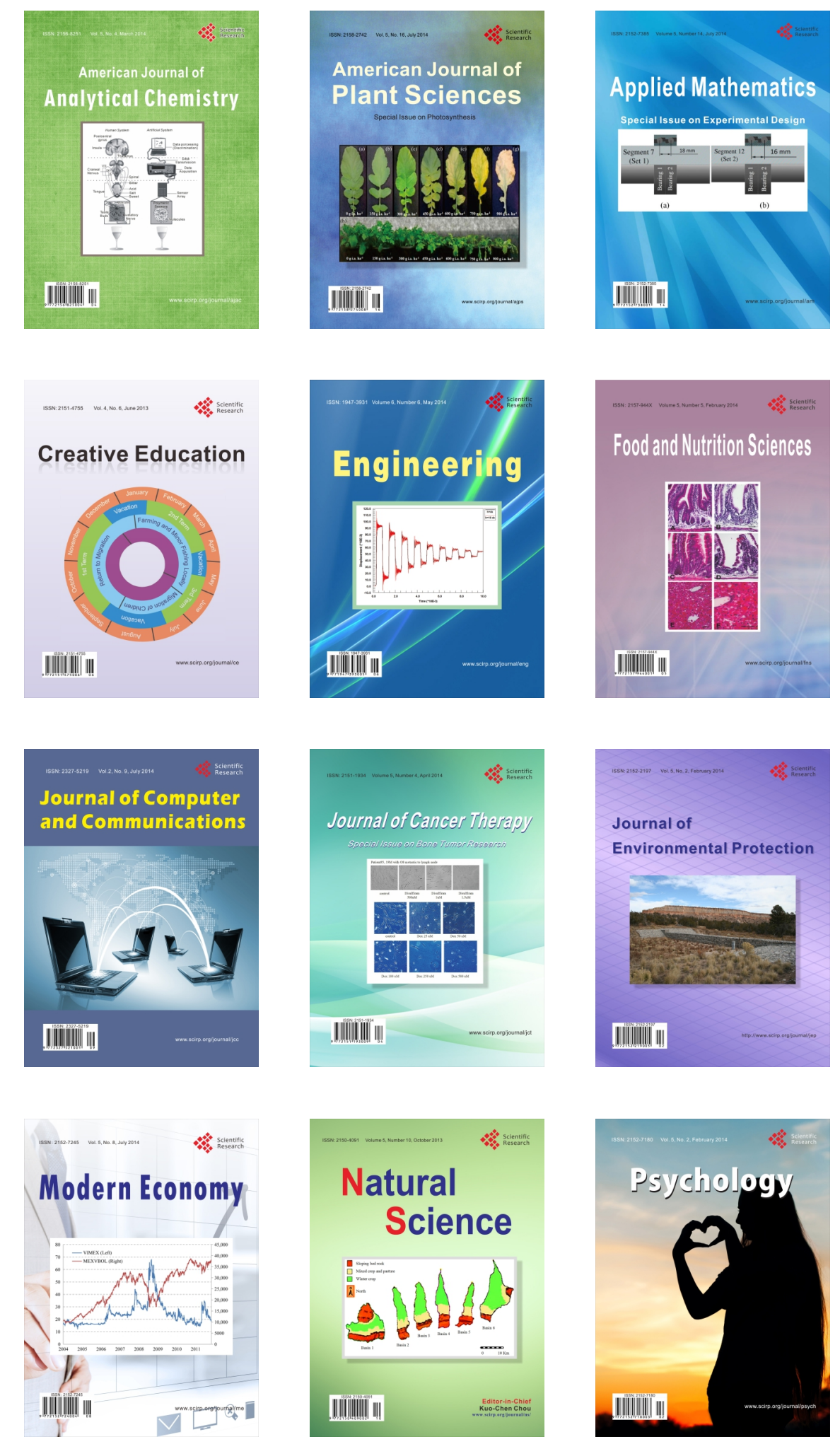